\title{
Intervenções em psicologia hospitalar durante a pandemia da COVID-19 no Brasil: uma revisão integrativa da literatura
}

\author{
Hospital psychology interventions during a COVID-19 pandemic in Brazil: an integrative literature \\ review
}

Intervenciones de psicología hospitalaria durante una pandemia de COVID-19 en Brasil: una revisión integradora de la literatura

\section{Resumo}

A pandemia da COVID-19 iniciou-se em dezembro de 2019 na China. Desde então, tem se alastrado por diversos países. Neste cenário pandêmico, a atuação dos(das) profissionais de psicologia têm sido imprescindível durante o tratamento. Este estudo teve como objetivo geral verificar as intervenções em psicologia hospitalar durante a pandemia da COVID-19 no Brasil. Para o alcance dos objetivos traçados, este estudo delimita-se como uma Revisão Integrativa da Literatura (RIL). A coleta de dados ocorreu no período de fevereiro a abril de 2021, nas bases de dados Google acadêmico, BVSPSI (Biblioteca Virtual de Psicologia), PePSIC (Periódicos Eletrônicos em Psicologia), LILACS (Literatura Latino-Americana e do Caribe em Ciências da Saúde) e Periódicos Capes. Os descritores e os operadores booleanos foram: psicologia hospitalar AND intervenções AND COVID-19 AND Brasil; psicologia AND hospital AND COVID-19 AND Brasil. Dessa forma, 22 estudos foram coletados. Após a leitura crítica dos títulos e resumos dos estudos 8 artigos foram selecionados e lidos na íntegra. Após este processo, todos foram utilizados na amostra final desta pesquisa. Os resultados demonstram que as intervenções foram voltadas aos pacientes e seus familiares como também aos profissionais de saúde. As principais intervenções foram: atendimento psicológico por telefone e presencial, atividades lúdicas, como também atividades psicoeducativas. Os objetivos deste estudo foram alcançados. Contudo, com uma limitação relacionada a ausência de produções acerca desta temática, sendo que, os poucos estudos publicados são do tipo relato de experiência. Neste sentido, considera-se relevante a realização de novos estudos.

Palavras-chave: COVID-19; Psicologia hospitalar; Intervenções.

\begin{abstract}
The COVID-19 pandemic began in December 2019 in China. Since then, it has spread to several countries. In this pandemic scenario, the role of psychology professionals has been essential during treatment. This study aimed to verify the interventions in hospital psychology during the COVID-19 pandemic in Brazil. In order to achieve the outlined objectives, this study is defined as an Integrative Literature Review (RIL). Data collection took place from February to April 2021, in Google academic databases, BVSPSI (Virtual Psychology Library), PePSIC (Electronic Journals in Psychology), LILACS (Latin American and Caribbean Literature in Health Sciences ) and Capes Periodicals. The descriptors and Boolean operators were: hospital psychology AND interventions AND COVID-19 AND Brazil; psychology AND hospital AND COVID-19 AND Brazil. Thus, 22 studies were collected. After critical reading of the titles and abstracts of the studies, 8 articles were selected and read in full. After this process, all were used in the final sample of this research. The results demonstrate that the interventions were aimed at patients and their families as well as health professionals. The main interventions were: psychological care by telephone and in person, recreational activities, as well as psychoeducational activities. The objectives of this study were achieved.
\end{abstract}


However, with a limitation related to the absence of productions on this theme, and the few published studies are of the experience report type. In this sense, it is considered relevant to carry out further studies.

Keywords: COVID-19; Hospital psychology; Interventions.

\section{Resumen}

La pandemia de COVID-19 comenzó en diciembre de 2019 en China. Desde entonces, se ha extendido a vários países. En este escenario pandémico, el papel de los profesionales de la psicología ha sido fundamental durante el tratamiento. Este estudio tuvo como objetivo verificar las intervenciones en psicología hospitalaria durante la pandemia de COVID-19 en Brasil. Para lograr los objetivos trazados, este estudio se define como una Revisión de Literatura Integrativa (RIL). La recolección de datos se realizó de febrero a abril de 2021, en las bases de datos académicas de Google, BVSPSI (Biblioteca Virtual de Psicología), PePSIC (Revistas Electrónicas de Psicología), LILACS (Literatura Latinoamericana y del Caribe en Ciencias de la Salud) y Publicaciones Periódicas de Capes. Los descriptores y operadores booleanos fueron: psicología hospitalaria Y intervenciones Y COVID-19 Y Brasil; psicología Y hospital Y COVID-19 Y Brasil. Así, se recopilaron 22 estudios. Después de la lectura crítica de los títulos y resúmenes de los estudios, se seleccionaron y leyeron en su totalidad 8 artículos. Después de este proceso, todos fueron utilizados en la muestra final de esta investigación. Los resultados demuestran que las intervenciones estaban dirigidas a los pacientes y sus familias, así como a los profesionales de la salud. Las principales intervenciones fueron: atención psicológica telefónica y presencial, actividades recreativas, así como atividades psicoeducativas. Se alcanzaron los objetivos de este estudio. Sin embargo, con una limitación relacionada con la ausencia de producciones sobre este tema, y los pocos estudios publicados son del tipo relato de experiencia. En este sentido, se considera relevante realizar más estudios.

Palabras clave: COVID-19; Psicología hospitalaria; Intervenciones.

\section{Introdução}

O surto de casos de COVID-19 iniciou-se em dezembro de 2019 na China. Desde então, tem se alastrado por diversos países e por isso, é considerado uma pandemia pela Organização Mundial da Saúde (OMS). Esta doença é causada pelo novo Coronavírus (SARS-CoV-2), e é transmitida através de gotículas de saliva ou espirro de pessoas infectadas. Além disso, a interação com estas pessoas e com ambientes infectados, também podem ser focos de transmissão (Rodrigues et al., 2020).

Com o aumento do número de casos da infecção e a ausência de vacina no início da pandemia, estratégias de contenção foram adotadas pelas autoridades governamentais em todo o mundo, como isolamento, utilização de quarentenas e de distanciamento social. O isolamento é uma medida que direciona as pessoas com o diagnóstico confirmado a permanecerem isoladas, sem interação com outras pessoas. A quarentena restringe a movimentação de pessoas que podem ser potenciais infectados por terem algum contato com o vírus. Já o distanciamento social busca evitar ao máximo o contato entre pessoas como, por exemplo, através do fechamento de locais públicos e privados, como escolas e comércio (Aquino et al., 2020).

Da mesma forma, os cuidados em saúde também necessitaram de mudanças por conta da pandemia da COVID-19, tais como a obrigatoriedade da utilização de equipamentos de proteção individual (EPI) pelos pacientes, realização de triagem dos pacientes com sintomas gripais nos serviços, onde pacientes com sintomas leves da COVID-19 poderiam manter-se isolados em domicílio, utilizando o teleatendimento em saúde, e, em caso necessários, lançando mão da internação hospitalar. Neste regime, o paciente deveria permanecer em uma enfermaria específica para tal demanda (COVID), com a devida sinalização sobre o risco biológico. Deste modo, diante de tantas mudanças, verifica-se a importância da atuação da equipe multiprofissional em saúde, no enfrentamento da COVID-19 (Freitas et al., 2020).

Nos últimos anos, a área da saúde tem sido marcada pelo aprimoramento de técnicas que possibilitam a melhora da qualidade de vida do indivíduo. Contudo, mesmo com tais avanços, a internação hospitalar ainda é um evento causador de dor e sofrimento, tanto nos pacientes, quanto em seus familiares. Neste cenário pandêmico, nota-se que a atuação dos profissionais de psicologia, enquanto membros constituintes das equipes multiprofissionais, é imprescindível durante o processo de diagnóstico, tratamento e de reabilitação do paciente, seja em regime de internação hospitalar ou não. (Ribeiro et al., 2020).

O setting terapêutico do(a) psicólogo(a) hospitalar é o hospital. Todavia, somente no Brasil, a atuação destes 
profissionais neste ambiente é denominada de psicologia hospitalar. Esta área foi ganhando forças a partir das mudanças em saúde nos atendimentos hospitalares no século XIX, buscando a integralidade da assistência em saúde, através da equipe multiprofissional. A profissional pioneira em psicologia hospitalar no Brasil foi a psicóloga Mathilde Neder, atuando na década de 50, no estado de São Paulo. Contudo, somente em 1962 a psicologia foi regulamentada enquanto uma profissão no Brasil. Em 1983, houve o I Encontro de Psicólogos Hospitalares. Desde então a área tem ganhado forças, e, somente em 20 de dezembro de 2000, o Conselho Federal de Psicologia (CFP), instituí título de especialistas em diversas áreas da psicologia, incluindo a Psicologias Hospitalar enquanto uma especialidade regulamentada no país (De Assis, 2020; Vieira e Waischunng, 2018).

Muitos autores, porém, defendem que a psicologia hospitalar faz parte da psicologia da saúde, sendo uma terminologia utilizada somente no Brasil. Contudo, entende-se, porém, que cada uma dessas especialidades possui um enfoque específico no que diz respeito a atuação do(a) psicólogo(a). A psicologia da saúde, através de técnicas psicológicas, busca a promoção, prevenção e o tratamento do adoecimento, objetivando verificar as variáveis que influenciam na relação do sujeito com a doença. Deste modo, o(a) psicólogo(a) da saúde pode atuar desde em hospitais, como em outras instituições de saúde (Ramos et al., 2018).

Segundo define a resolução no 013/2007 do Conselho Federal de Psicologia (CFP), o profissional especialista em psicologia hospitalar pode atuar tanto no setor secundário, quanto no terciário da saúde. A intervenção deve ser voltada a todos os envolvidos no processo de saúde (usuários/pacientes, profissionais e familiares), podendo atuar em diversos setores, como em Unidade de Terapia Intensiva (UTI) e enfermarias em geral.

Acerca do objetivo da psicologia hospitalar, Simonetti (2016) afirma que a sua atuação é voltada para a subjetividade do paciente, a partir da escuta psicológica, auxiliando este sujeito a lidar com seu adoecimento, entrando em contato com seus sintomas, mesmo quando este está impossibilitado de falar. Logo, entende-se que a psicologia não busca a cura das doenças, como a medicina. Mas volta-se para algo mais profundo, que seria intervir diante das consequências causadas pela mesma, tanto no paciente, quanto na família e nos profissionais de saúde.

As Referências Técnicas para a atuação de psicólogos(as) nos serviços hospitalares do SUS, apontam que o profissional de psicologia está inserido em diversos processos, como o acolhimento do indivíduo ou grupo, este processo pode ser realizado pela equipe multiprofissional a partir da escuta do paciente ou de seus familiares quando buscam assistência em saúde, quando é realizado pelo(a) profissional de psicologia esta intervenção é utilizada também como estratégia de criação de vínculo para a facilitação das demais etapas do processo terapêutico e acompanhamento, serviço este no qual o(a) psicólogo(a) proporciona a escuta qualificada ao paciente e se faz presente durante todo o tratamento do mesmo (CFP, 2019).

Dessa forma, levando em consideração a pandemia da COVID-19 e a atuação do(a) psicólogo(a) hospitalar, este estudo teve como objetivo geral verificar as intervenções em psicologia hospitalar durante a pandemia da COVID-19 no Brasil, tendo ainda como objetivos específicos descrever tais intervenções, demonstrar as adaptações nos atendimentos psicológicos hospitalares e identificar as áreas de atuação dos profissionais de psicologia durante a pandemia, através da realização de uma Revisão Integrativa de Literatura - RIL.

\section{Metodologia}

Este estudo caracteriza-se por uma revisão integrativa da literatura (RIL). A organização desta pesquisa baseou-se em Mendes, Siqueira e Galvão (2008), no qual afirmam que a revisão integrativa pode ser organizada a partir das seguintes etapas: formulação da questão norteadora, busca na literatura, avaliação crítica dos estudos, coleta de dados, análise e apresentação dos dados, interpretação dos dados e aprimoramento e atualização da revisão. A pergunta norteadora que se busca responder neste 
estudo é: Quais as intervenções em psicologia hospitalar realizadas durante a pandemia da COVID-19?

A coleta de dados ocorreu no período de fevereiro a abril de 2021, nas bases de dados Google acadêmico, BVSPSI (Biblioteca Virtual de Psicologia), PePSIC (Periódicos Eletrônicos em Psicologia), LILACS (Literatura Latino-Americana e do Caribe em Ciências da Saúde) e Periódicos Capes. Como critérios de inclusão, foram utilizadas pesquisas de 2019 a 2021 , publicadas em língua portuguesa e que tivessem relação com o tema proposto. Já como critérios de exclusão, foram excluídos artigos que não foram publicados entre os anos de 2019 e 2021, que não fossem escritos na língua portuguesa e que não tivessem relação com o tema. Os descritores e os operadores booleanos foram: psicologia hospitalar AND intervenções AND COVID-19 AND Brasil; psicologia AND hospital AND COVID-19 AND Brasil.

Tabela 1. Publicações disponíveis no período de 2019 a 2021 nas bases de dados.

\begin{tabular}{|c|c|c|c|}
\hline $\begin{array}{l}\text { Dase de } \\
\text { dados }\end{array}$ & $\begin{array}{c}\text { Psicologia Hospitalar AND } \\
\text { Intervenções AND COVID-19 } \\
\text { AND Brasil }\end{array}$ & $\begin{array}{c}\text { Psicologia AND Hospital AND } \\
\text { COVID-19 AND Brasil }\end{array}$ & TOTAL \\
\hline Google Acadêmico & 1620 estudos & 0 estudos & 5620 estudos \\
\hline BVSPSI & 0 estudos & 0 estudos & 0 estudos \\
\hline PEPSIC & 0 estudos & 0 estudos & 0 estudos \\
\hline LILACS & 0 estudos & 2 estudos & 2 estudos \\
\hline Periódicos Capes & 3 estudos & 4 estudos & 7 estudos \\
\hline TOTAL & 1623 estudos & 4006 estudos & 5629 estudos \\
\hline
\end{tabular}

Fonte: Autores.

Ao utilizar os descritores Psicologia Hospitalar AND Intervenções AND COVID-19 AND Brasil, na base de dados do Google acadêmico foram encontrados 1620 estudos, na BVSPSI, PEPSIC e LILACS não foi encontrado nenhum estudo, já no Periódicos Capes foram encontrados apenas 3 estudos. Totalizando 1623 estudos.

Na utilização dos descritores Psicologia AND Hospital AND COVID-19 AND Brasil, foram encontrados na base de dados do Google Acadêmico 4000 estudos, na BVSPSI e PEPSIC não foi encontrado nenhum estudo, na LILAS encontrou-se 2 estudos e no Periódicos Capes foram encontrados 4 estudos, totalizando assim 4006 estudos. Ao todo, foram encontrados 5629 estudos utilizando os 2 descritores nas 5 bases de dados citadas. Contudo, apenas 22 estudos foram coletados para serem avaliados, por estarem de acordo com os critérios de inclusão e exclusão determinados pela pesquisa.

\section{Resultados e Discussão}

Foram coletadas um total de 22 pesquisas. Após a leitura crítica dos títulos e resumos dos estudos, 14 foram excluídos, restando apenas 8 estudos, que foram lidos integralmente e utilizados na amostra final desta pesquisa. Na Tabela 2 , estão dispostos os estudos que foram selecionados e utilizados na amostra final desta pesquisa. 
Tabela 2. Disposição dos títulos, autores, ano da publicação, periódico e tipo de estudo utilizados.

\begin{tabular}{|c|c|c|c|c|}
\hline $\mathbf{N}^{\mathbf{o}}$ & Título & $\begin{array}{l}\text { Autores/ Ano da } \\
\text { publicação }\end{array}$ & Periódico & Tipo de Estudo \\
\hline 1 & $\begin{array}{l}\text { Psicanálise aplicada ao contexto hospitalar: } \\
\text { intervenções em tempos de pandemia Covid- } \\
19\end{array}$ & Almendra et al./ 2019 & Revista aSEPHallus & $\begin{array}{l}\text { Relato de } \\
\text { experiência }\end{array}$ \\
\hline 2 & $\begin{array}{l}\text { Quando o" fique em casa" não era uma } \\
\text { opção: os bastidores e os relatos das } \\
\text { experiências dos profissionais de saúde no } \\
\text { front de combate à pandemia da COVID-19. }\end{array}$ & Dias et al./2019 & Revista aSEPHallus & $\begin{array}{l}\text { Relato de } \\
\text { experiência }\end{array}$ \\
\hline 3 & $\begin{array}{l}\text { A esperança venceu o medo: psicologia } \\
\text { hospitalar na crise do COVID-19. }\end{array}$ & Lima et al./2020. & $\begin{array}{l}\text { Revista Científica } \\
\text { da Escola de Saúde } \\
\text { Pública do Ceará }\end{array}$ & $\begin{array}{l}\text { Relato de } \\
\text { Experiência }\end{array}$ \\
\hline 4 & $\begin{array}{l}\text { A inserção de duas psicólogas residentes em } \\
\text { tempos de COVID-19 }\end{array}$ & Silva e Lima/ 2020 & $\begin{array}{c}\text { Revista Científica } \\
\text { da Escola de Saúde } \\
\text { Pública do Ceará }\end{array}$ & $\begin{array}{l}\text { Relato de } \\
\text { Experiência }\end{array}$ \\
\hline 5 & $\begin{array}{c}\text { A morte na Pandemia COVID-19: } \\
\text { Articulando a minha experiência da prática } \\
\text { psicológica no Hospital com a Teoria da } \\
\text { Gestalt-terapia }\end{array}$ & Lessa/2020 & $\begin{array}{l}\text { Revista IGT na } \\
\text { Rede }\end{array}$ & Estudo de Caso \\
\hline 6 & $\begin{array}{c}\text { Humanização no hospital: atuação da } \\
\text { psicologia na COVID-19 }\end{array}$ & Catunda et al./ 2020 & $\begin{array}{c}\text { Revista Científica } \\
\text { da Escola de Saúde } \\
\text { Pública do Ceará }\end{array}$ & $\begin{array}{l}\text { Relato de } \\
\text { Experiência }\end{array}$ \\
\hline 7 & $\begin{array}{l}\text { Como os hospitais universitários estão } \\
\text { enfrentando a pandemia de COVID-19 no } \\
\text { Brasil? }\end{array}$ & Santos et al./2020 & $\begin{array}{l}\text { Acta Paulista de } \\
\text { Enfermagem }\end{array}$ & $\begin{array}{c}\text { Estudo } \\
\text { Documental }\end{array}$ \\
\hline 8 & $\begin{array}{l}\text { Atendimento psicológico aos pacientes em } \\
\text { Ventilação Mecânica Invasiva: relato de } \\
\text { experiência em uma Unidade de Terapia } \\
\text { Intensiva na pandemia de COVID-19 }\end{array}$ & Souza/2021 & $\begin{array}{l}\text { Brazilian Journal of } \\
\text { Development }\end{array}$ & $\begin{array}{l}\text { Relato de } \\
\text { Experiência }\end{array}$ \\
\hline
\end{tabular}

Fonte: Autores.

Dentre os artigos selecionados para a amostra final deste estudo, 75\% (artigos 1, 2, 3, 4, 6 e 8) são pesquisas do tipo de relato de experiência, 12,5\% (artigo 5) é estudo de caso e os outros 12,5\% (artigo 7) é estudo documental. No que se refere ao ano de publicação, 62,5\% (artigos 3, 4, 5, 6 e 7) foram publicados em 2020, 25\% (artigos 1 e 2) foram publicados em 2019 e apenas 12,5\% (artigo 8) foi publicado em 2021.

Acerca dos hospitais no qual os estudos foram realizados, 50\% deles (artigos 4, 5, 7 e 8) foram realizados em hospitais públicos, 37,5\% (artigos 1, 2 e 6) foram realizados em hospitais privados e 12,5\% (artigo 3) foi realizado em hospital de caráter filantrópico. Além disso, 2 regiões do Brasil publicaram mais estudos acerca desta temática, a região nordeste com 50\% (artigos 3, 4, 6 e 8), sendo 3 realizados na cidade de Fortaleza e 1 na cidade de Pernambuco; e a região sudeste com 37,5\% (artigos 1, 2 e 5), todos na cidade do Rio de Janeiro. Contudo, 12,5\% (artigo 7) foi um estudo documental, que cita hospitais universitários de vários estados do Brasil.

No que se refere ao direcionamento dado pelas principais intervenções da Psicologia Hospitalar citadas nas pesquisas durante a pandemia da COVID-19, 25\% foram voltadas aos profissionais, pacientes e familiares (artigos 3 e 8 ); outros $25 \%$ (artigos 4 e 6) citaram intervenções voltadas apenas aos pacientes; 12,5\% (artigo 5) descreveu intervenções voltadas aos familiares; $12,5 \%$ (artigo 1) englobou os familiares e pacientes, excluindo profissionais de saúde; $12,5 \%$ (artigo 2) optou por descrever intervenções destinadas aos profissionais e pacientes; e 12,5\% (artigo 7) citou intervenções direcionadas as equipes de saúde apenas. Figura 2. 
Os resultados confirmam o enfoque das intervenções realizadas por profissionais da psicologia hospitalar no decorrer da pandemia da COVID 19 no país, já descritos na literatura. Esses dados vêm ao encontro do que defende Andrade (2018), a respeito do papel do profissional da psicologia hospitalar estar centrado em torno da relação da "tríade", que engloba paciente, profissionais da saúde e familiares. $\mathrm{O}(\mathrm{A})$ psicólogo(a) no ambiente hospitalar tem como função facilitar a comunicação entre essa tríade e assim possibilitar com que todas pessoas inseridas neste processo, possam lidar de modo mais saudável com suas emoções e com as experiências vivenciadas na hospitalização.

A partir das informações coletadas, os resultados dessa revisão integrativa de literatura foram categorizados conforme o enfoque das intervenções psicológicas realizadas durante a pandemia de COVID 19, em: Intervenções voltadas aos pacientes, intervenções destinadas aos familiares e intervenções direcionadas aos profissionais da saúde.

Acerca das intervenções destinadas aos pacientes internados, observou-se que o atendimento psicológico enquanto modalidade de intervenção sofreu adaptações diante do contexto pandêmico. Verificou-se que este tipo de atendimento foi realizado a partir de ligação telefônica para todos os pacientes internados, segundo o estudo de Dias et al. (2019). Já na pesquisa realizada por Lima et al. (2020), este atendimento foi realizado também via ligação telefônica, contudo, apenas para pacientes que estavam em regime de isolamento, devido o diagnóstico por COVID-19 confirmado ou suspeito. Os demais pacientes internados eram atendidos presencialmente, estando o(a) psicólogo(a) devidamente paramentado com os Equipamentos de Proteção Individual (EPI's) apropriados. No relato de experiência de Silva e Lima (2020), os pacientes com confirmação e suspeita de COVID-19, foram atendidos presencialmente pelos profissionais da psicologia, que também precisaram utilizar EPI's como máscara, aventais e luvas. Ainda neste mesmo estudo, a técnica psicológica utilizada nos atendimentos foi psicoterapia breve, através da escuta qualificada.

Em 2018, o Conselho Federal de Psicologia (CFP) emitiu a resolução ${ }^{\circ} 11$ de 11 de maio de 2018, que regulamenta a utilização das tecnologias de comunicação para atendimentos psicológicos. Nela, em seu artigo $7^{\circ}$, era vedado o atendimento psicológico a pacientes em casos de urgência e emergência. Contudo, em 2020 o CFP publicou a resolução $\mathrm{n}^{\circ} 4$, de 11 de março de 2020, acerca dos atendimentos psicológicos através das tecnologias de informação durante a pandemia da COVID19 , no qual suspendeu alguns artigos da resolução $n^{\circ} 11$ de maio de 2018 , como o artigo $7^{\circ}$, permitindo que pacientes em casos de urgência e emergência pudessem ser atendidos por essas tecnologias.

Ainda a respeito das intervenções psicológicas, o estudo de Souza (2021) acerca do atendimento psicológico a pacientes em Ventilação Mecânica Invasiva (VMI) em uma Unidade de Terapia Intensiva de COVID-19, descreveu que, os(as) psicólogos(as) lançaram mão de estratégias não verbais durante as intervenções para os pacientes que estavam em VMI, sem sedação. No mesmo estudo, também foi descrita a utilização de vídeo chamada ou de chamada de voz para a comunicação dos pacientes com seus familiares. Contudo, por conta da limitação de muitos pacientes em relação a comunicação verbal, foi pedido aos familiares que gravassem vídeos ou áudios com mensagens de conforto, para serem reproduzidas posteriormente. Esta mesma intervenção também foi utilizada com pacientes que estavam sedados, quando se percebeu que, quando o vídeo ou o áudio foi reproduzido aos pacientes, notou-se a manifestação de reações fisiológicas como o aumento dos batimentos cardíacos. Além disso, também foram utilizadas leituras de cartas escritas pelos familiares aos pacientes em regime de internação hospitalar.

De acordo com Pesoli e Nascimento (2018), a dificuldade do paciente em se comunicar, seja por estar questões fisiológicas como traumatismo ou até mesmo por conta de algum procedimento do tratamento hospitalar, pode contribuir para a vivência de emoções negativas, como também frustração durante este processo. Diante disso, faz-se importante haver estratégias de comunicação alternativa. Simonetti (2016), afirma que o papel do(a) psicólogo(a) no atendimento a pacientes com dificuldades de comunicação verbal é buscar instrumentos alternativos para que estes consigam se expressar, como 
através da criação de bilhetes, e da utilização de gestos, por exemplo. Contudo, o mesmo autor ressalta que o papel desta comunicação não se restringe somente a transmitir informações, e sim a potencializar a relação com o paciente.

Os estudos demonstraram também a utilização de outras estratégias interventivas durante e após a internação hospitalar, como por exemplo, o uso de materiais psicoterapêuticos pelos profissionais de psicologia. Dessa forma, em um dos serviços citados, foi criado o "livro da afetividade", que continha atividades lúdicas, reflexões e também informações acerca do autocuidado e manejo de ansiedade, devido a condição de isolamento e impossibilidade de permanecer em companhia de familiar. Outra estratégia utilizada, tratava do momento da alta hospitalar, quando ao paciente era entregue a chamada "carta da gratidão", em que continha um agradecimento aos pacientes por permitirem serem cuidados pelos profissionais de saúde daquele hospital. Além disso, nesta carta também haviam orientações acerca da importância da continuidade do autocuidado, como conteúdo para ajudá-lo a ressignificar suas vivências durante o período de hospitalização (Lima et al., 2020).

Segundo Silva e Silva (2012), a utilização de materiais lúdicos durante a hospitalização tem como característica o cuidado humanizado e auxilia o paciente no seu processo de recuperação. A característica principal deste instrumento é proporcionar sensações de bem estar, potencializando a criatividade e proporcionando o divertimento ao paciente. O lúdico auxilia ainda o paciente a lidar com suas emoções, bem como com o sofrimento vivenciado a partir da internação. Além, disso facilita na relação terapêutica entre o paciente e os profissionais de saúde.

Devido as restrições causadas pela pandemia da COVID-19, as visitas presenciais aos pacientes foram suspensas. Contudo, alguns serviços de saúde organizaram visitas virtuais, através da utilização de tablets nos diversos setores dos hospitais. Assim, os(as) psicólogos(as) ficavam responsáveis por verificar com as equipes multiprofissionais qual o paciente que estava apto para receber a "visita", entravam em contato com os seus familiares, para agendar o melhor horário para que esta atividade ocorresse, avaliando ainda quem da família poderia estar presente neste momento de encontro virtual (Catunda et al.,2020).

Com a pandemia da COVID-19, a interação social precisou sofre alterações também por conta do alto nível e contágio da doença. Os hospitais necessitaram suspender as visitas presenciais o que pode afetar a saúde mental dos pacientes e aumentar a sensação de solidão. Como uma forma de amenizar o sofrimento do paciente e da família, muitos profissionais de saúde utilizaram de seus próprios aparelhos tecnológicos para que a comunicação entre o paciente e seus familiares acontecesse. Neste sentido, a partir de diversas experiências observadas pelos profissionais, nota-se o quanto que esta interação possibilitou aos pacientes e seus familiares, apoiarem uns aos outros durante o processo de hospitalização (Rios et al., 2020).

Nos estudos de Aumendra et al. (2019) Dias et al. (2019), Lima et al. (2020) e Souza (2021), os atendimentos psicológicos aos familiares foram oferecidos através de ligações telefônicas. Especificamente no estudo de Aumendra et al. (2019), os atendimentos aconteciam no período da tarde, a todos os familiares dos pacientes que estavam em isolamento, quando eram repassadas informações não-médicas, como uma estratégia de ajudar a família a lidar com a ansiedade em relação ao boletim médico.

Dias et al. (2019), relatam que os profissionais de psicologia davam o suporte psicológico aos familiares dos pacientes, de modo especial aos que estavam internados em UTI. Durante as ligações, os profissionais de psicologia não somente davam o suporte psicológico. Contudo, precisavam intervir para que os familiares compreendessem as restrições em relação as visitas e também as informações dadas pelos médicos, que muitas vezes não eram compreendidas pela família.

Lima et al. (2020), descrevem que os(as) psicólogos(as) entravam primeiramente em contato com um dos familiares e explicavam qual o objetivo do serviço ofertado. Caso fosse necessário, o atendimento poderia ser disponibilizado também a outros familiares, através do número de telefone fornecido, aprazando um horário para esta prestação de serviços psicológicos. Grincenkov (2020), afirma que o papel da psicologia neste período de pandemia é auxiliar tanto o paciente quanto aos 
familiares a lidarem com a hospitalização e com as restrições por conta da letalidade da COVID-19. Além disso, a intervenção psicológica deve ajudar o paciente e seus familiares a lidarem com pensamentos negativos, que podem dificultar a vivência e a adaptação dos mesmos em relação ao processo de internação.

Conforme descrito por Lessa (2020) em seu estudo de caso, a profissional da psicologia hospital citada, deu suporte psicológico presencial aos familiares após as notícias de óbito, bem como interveio através de gestos, como o toque nas costas ou na mão. Já no estudo de Lima et al. (2020), os profissionais de psicologia elaboraram a chamada "carta da esperança", que era entregue aos familiares que estavam vivenciando o luto, e continha informações psicoeducativas para ajudarem a lidar com o luto.

Nesse sentido, Gobbi (2020), afirma que a psicologia na comunicação de más notícias pode atuar antes, durante ou após este processo, enfatizando que a comunicação em si deve ser realizada pelos profissionais de medicina. Após ser repassada a informação, o(a) psicólogo(a) deve intervir através do acolhimento e ajudando os familiares a lidarem com suas emoções diante da notícia recebida.

De acordo com Dias et al. (2019), os profissionais da saúde também receberam atendimento psicológico, tanto de modo remoto, quanto presencial. Além disso, os profissionais de psicologia também elaboraram uma cartilha com informações para ajuda-los em relação autocuidado da saúde mental durante a pandemia da COVID-19.

Em sua pesquisa, Lima et al. (2020), descreve também que foi disponibilizado atendimento psicológico aos profissionais da saúde, de modo on line, através do plantão psicológico. Contudo, outras estratégias também foram elaboradas pelos(as) psicólogos(as), como a criação de vídeos com informações psicoeducativas, no qual ensinavam, por exemplo, técnicas de relaxamento, estratégias para os profissionais lidarem com o isolamento social, etc. Foi criado ainda o mural da esperança, que continha frases reflexivas, desafios de autocuidado, fotos dos funcionários, registros fotográficos das atividades realizadas com os pacientes, depoimentos de familiares, pacientes e de funcionário, notícias positivas em relação ao enfrentamento da pandemia, o número de pessoas que receberam alta e desenhos de crianças filhos dos funcionários. Sobre "o desafio do autocuidado" o estudo descreve que os profissionais da equipe de saúde eram estimulados a praticarem o autocuidado durante quatro semanas.

Já no estudo de Souza (2021), os profissionais de psicologia realizaram atividades psicoeducativas com os profissionais da saúde, no sentido de ajudá-los a compreender a importância do atendimento humanizado como, por exemplo, se apresentar e informar a todos os pacientes atendidos, até mesmo os que estão inconscientes, explicando os procedimentos que serão realizados. No manual de atenção psicológica nos hospitais em tempos e combate ao COVID-19, Serafim e Nunes (2020) afirmam que o atendimento psicológico nos hospitais é voltado não somente para o paciente, mas também para todos os profissionais que compõe a equipe multidisciplinar e até mesmo para a própria equipe de psicologia. Além disso, Teixeira et al. (2020) discorrem acerca dos diversos impactos da pandemia na saúde mental dos profissionais de saúde como pressão psicológica, medo de serem infectados, isolamento social, estresse entre outros.

Segundo a cartilha de orientações aos(as) psicólogos(as) hospitalares da Fundação Oswaldo Cruz (2020), os profissionais de psicologia deveriam organizar atividades para a prevenção e promoção da saúde mental de todos os profissionais dos hospitais informando os atendimentos psicológicos oferecidos pela instituição, como também divulgando práticas de autocuidado.

\section{Conclusão}

Os resultados apresentados neste estudo indicam que as intervenções do(a) psicólogo(a) hospitalar não deve ser focado somente no paciente, mas também nos demais indivíduos envolvidos no processo de internação: os familiares e os 
profissionais de saúde. Verificou-se que, assim como a sociedade em geral, os atendimentos em saúde necessitaram passar por adaptações diante do contexto de pandemia. A escuta no ambiente hospitalar, o principal instrumento do(a) psicólogo(a), em muitos casos, não pôde ser realizado presencialmente e assim foi intermediada através de aparelhos tecnológicos a alguns pacientes e aos demais indivíduos envolvidos neste processo.

Nota-se também, o importante papel da psicologia no atendimento a pacientes que apresentaram limitação em sua comunicação, bem como a criação de materiais terapêuticos e a organização de visitas virtuais como estratégia de amenizar o sofrimento psicológico tanto dos pacientes, quanto dos familiares e profissionais de saúde. Além disso, durante a comunicação de notícias difíceis, a psicologia também se fez presente através da facilitação do entendimento dos familiares acerca do diagnóstico, como também no suporte psicológico disponibilizado aos mesmos após as comunicações dos óbitos.

O sofrimento psicológico causado pelo adoecimento e pela internação hospitalar já era percebido nos pacientes, nos seus familiares e nos profissionais de saúde antes da pandemia. Todavia, com atual contexto pandêmico, diante de tantas incertezas e mudanças repentinas, este sofrimento tem sido potencializado. Assim, verifica-se o relevante papel dos(as) profissionais de psicologia no ambiente hospitalar. Por isso, espera-se através deste estudo contribuir com o fomento da pesquisa científica nesta área, possibilitar uma maior valorização desta categoria, ressaltar a importância da busca constate de atualização dos conhecimentos destes profissionais, incentivar o autocuidado dos mesmos, já que precisam lidar com o sofrimento dos outros, como também ressaltar a necessidade de haver um maior investimento governamental no cuidado com a saúde mental da população.

Por fim, mesmo que os objetivos desta pesquisa tenham sido alcançados, observou-se a escassez de estudos acerca da temática abordada. Por isso, faz-se necessário a realização de novas pesquisas. Como sugestão para as futuras pesquisas, é interessante que possam ser pesquisas de campo, já que nota-se o grande número de publicações de relatos de experiência.

\section{Referências}

Almendra, F. S. R., Santos, T. C. D., Moreira, M. I. R., \& Castro, M. G. S. R. D. (2019). Psicanálise aplicada ao contexto hospitalar: intervenções em tempos de pandemia Covid-19. aSEPHallus, 92-102.

Aquino, E. M., Silveira, I. H., Pescarini, J. M., Aquino, R., Souza-Filho, J. A. D., Rocha, A. D. S., ... \& Lima, R. T. D. R. S. (2020). Medidas de distanciamento social no controle da pandemia de COVID-19: potenciais impactos e desafios no Brasil. Ciência \& Saúde Coletiva, $25,2423-2446$.

Andrade, D. S. (2017). O papel do psicólogo no hospital na visão dos profissionais de saúde.

Catunda, M. L., dos Santos, L. N. A., de Souza, C. B., Porto, A. B., Nardino, F., Lima, M. E. G., \& de Araújo, V. S. (2020). Humanização no hospital: atuações da psicologia na COVID-19: humanization in the hospital: psychology performance in COVID-19. Cadernos ESP-Revista Científica da Escola de Saúde Pública do Ceará, 14(1), 143-147.

De Assis, F. E. (2020). A atuação da psicologia hospitalar, breve histórico e seu processo de formação no Brasil. Psicologia Argumento, 37(98), 501-512.

Dias, M. D. S. F. M., Santos, T. C. D., Pereira, F. R. C., Rodrigues, D. R. D. S., \& Costa, E. A. D. S. D. G. (2019). Quando o" fique em casa" não era uma opção: os bastidores e os relatos das experiências dos profissionais de saúde no front de combate à pandemia da COVID-19. aSEPHallus, 118-128.

Freitas, R., de Oliveira, L. A. F., da Costa Rosa, K. S., Borsatto, A. Z., Sampaio, S. G. D. S. M., Sales, B. R., ... \& de Oliveira, L. C. (2020). Cuidados paliativos em pacientes com câncer avançado e Covid-19. Revista Brasileira de Cancerologia, 66(TemaAtual).

Gobbi, M. B. (2020). Comunicação de más notícias: um olhar da psicologia. Diaphora, 9(1), 66-69.

Grincekov F. R. (2020). A Psicologia Hospitalar e da Saúde no enfrentamento do coronavírus: necessidade e proposta de atuação. Hu Revista, (46), 1-2.

Lima, M. J. V., Gonçalves, E. F. L. M., Vasconcelos, A. B. L. P., de Abreu, A. R. S., \& Mendonça, S. M. (2020). A esperança venceu o medo: psicologia hospitalar na crise do COVID-19.: hope overcome fear: hospital psychology in the COVID-19 crisis. Cadernos ESP-Revista Científica da Escola de Saúde Pública do Ceará, 14(1), 100-108.

Lessa, A. S. (2020). A morte na Pandemia COVID-19: Articulando a experiência da prática psicológica no Hospital com a Teoria da Gestalt-terapia Death in the COVID-19 Pandemic: Articulating my experience of psychological practice in the Hospital with the Theory of Gestalt T. IGT na Rede ISSN 1807-2526, 17(32), Brasil-Brasil.

Mendes, K. D. S., Silveira, R. C. D. C. P., \& Galvão, C. M. (2008). Revisão integrativa: método de pesquisa para a incorporação de evidências na saúde e na enfermagem. Texto \& contexto-enfermagem, 17, 758-764. 
Research, Society and Development, v. 10, n. 12, e332101220288, 2021

(CC BY 4.0) | ISSN 2525-3409 | DOI: http://dx.doi.org/10.33448/rsd-v10i12.20288

Orientações as/aos psicólogas(os) hospitalares (2020). Brasília: Fiocruz.

Pelosi, M. B., \& Nascimento, J. S. (2018). Uso de recursos de comunicação alternativa para internação hospitalar: percepção de pacientes e de terapeutas ocupacionais/Use of alternative communication resources for hospital intervention: perception of patients and occupational therapists. Cadernos Brasileiros de Terapia Ocupacional, 26(1).

Rios, I. C., Carvalho, R. T. D., Ruffini, V. M. T., Montal, A. C., Harima, L. S., Crispim, D. H., ... \& Bonfa, E. S. D. D. O. (2020). Virtual visits to inpatients by their loved ones during COVID-19. Clinics, 75.

Ramos, C. S., Almeida, M. L., Brito, S. S., \& Campos Bahia Moscon, D. (2018). Os desafios que os psicólogos hospitalares encontram ao longo de sua atuação. Seminário Estudantil de Produção Acadêmica, 16.

Ribeiro, C. B. N., de Souza, D. O., Horst, E. P. C., Alves, E. C., Zazatt, T. D. A. L., \& Fitaroni, J. (2020). A atuação do psicólogo nos cuidados paliativos. TCC-Psicologia.

Rodrigues, JVS, Cardoso, AJ, Gualberto, LGC, Monteiro, JD, de Lima, BJM, \& Cruz, CRP (2020). Estágio supervisionado em Psicologia da Saúde durante a pandemia da COVID-19. Research, Society and Development, 9 (9), e680997580-e680997580.

Referências técnicas para a atuação de psicólogos(as) nos serviços hospitalares do SUS (2019). Brasília: Conselho Federal de Psicologia.

Resolução $n^{\circ}$ 013/2007 (2007). Institui a Consolidação das Resoluções relativas ao Título Profissional de Especialista em Psicologia e dispõe sobre normas e procedimentos para seu registro. Brasília: Conselho Federal de Psicologia.

Resolução $\mathrm{n}^{\circ} 11$ (2018). Regulamenta a prestação de serviços psicológicos realizados por meios de tecnologias da informação de serviços psicológicos realizados por meios de tecnologias da informação e da comunicação e revoga a Resolução CFP N 11/2012. Brasília: Conselho Federal de Psicologia.

Resolução $n^{\circ} 4$ (2020). Dispõe sobre regulamentação de serviços psicológicos prestados por meio de Tecnologia da Informação e da Comunicação durante a pandemia do COVID-19. Brasília: Conselho Federal de Psicologia.

Serafim, R. S., Do Bú, E., \& Nunes, A. L. (2020). Manual de diretrizes para atenção psicológica nos hospitais em tempos de combate ao Covid-19. Revista Saúde \& ciência Online, $9(1)$.

Santos, J. L. G. D., Lanzoni, G. M. D. M., Costa, M. F. B. N. A. D., Debetio, J. O., Sousa, L. P. D., Santos, L. S. D., ... \& Mello, A. L. S. F. D. (2020). Como os hospitais universitários estão enfrentando a pandemia de COVID-19 no Brasil?. Acta Paulista de Enfermagem, 33.

Silva, A. C. M., \& da Silva, M. A. (2012). As contribuições da arte lúdica do restabelecimento da saúde humana. Revista EVS-Revista de Ciências Ambientais e Saúde, 39(4), 469-480.

Souza, S. A. R. (2021). Atendimento psicológico aos pacientes em Ventilação Mecânica Invasiva: relato de experiência em uma Unidade de Terapia Intensiva na pandemia de COVID-19. Brazilian Journal of Development, 7(2), 20265-20275.

Silva, K. C. L., \& Lima, M. E. G. (2020). A inserção de duas psicólogas residentes em tempos de COVID-19. Cadernos ESP-Revista Científica da Escola de Saúde Pública do Ceará, 14(1), 95-99.

Simonetti, A. (2006). Manual de psicologia hospitalar. Casa do psicólogo.

Teixeira, C. F. D. S., Soares, C. M., Souza, E. A., Lisboa, E. S., Pinto, I. C. D. M., Andrade, L. R. D., \& Espiridião, M. A. (2020). A saúde dos profissionais de saúde no enfrentamento da pandemia de Covid-19. Ciência \& Saúde Coletiva, 25, 3465-3474.

Vieira, A. G., \& Waischunng, C. D. (2018). A atuação do psicólogo hospitalar em Unidades de Terapia Intensiva: a atenção prestada ao paciente, familiares e equipe, uma revisão da literatura. Revista da $S B P H, 21(1), 132-153$. 